\title{
Universal Wire Grid for Implant Placement in Three Dimensions
}

\author{
Narendra S Sharma, Sunita S Shrivastav, Pushpa V Hazarey, RH Kamble, Preethi N Sharma
}

\begin{abstract}
Implant used for skeletal anchorage offers clinical advantage of being smaller in size, ease of insertion and removal, the ability to load forces immediately and rapid healing. The risk of injury to dental roots during placement is one of the greatest concerns with implant especially when they are inserted between teeth.

Placement of implant to close to the root can also result in insufficient bone remodeling around the screw and transmission of occlusal forces through the teeth to the screw which can lead to implant failure. Therefore it is important to select insertion site carefully using clinical and radiographic evaluation. Many techniques have been used to facilitate safe placement of implant. Some or the others may be inconsistent in different radiographic view.

This article introduces a three-dimensional (3D) wire grid which is simple, reliable and accurate for placing implant in a single step which improves the insertion success rate.
\end{abstract}

Keywords: Microimplant, 3D acrylic wire grid, Skeletal anchorage, Orthodontic mini-implants.

How to cite this article: Sharma NS, Shrivastav SS, Hazarey PV, Kamble RH, Sharma PN. Universal Wire Grid for Implant Placement in Three Dimensions. World J Dent 2013;4(1): 74-76.

\section{Source of support: Nil}

Conflict of interest: None declared

\section{INTRODUCTION}

Recently with the advent of implant supported treatment modalities the envelope of correction amenable to conventional mechanics has expanded. Popular among the implants are microscrew implants as their size permits placement in areas previously inaccessible. However, like brackets accurate positioning of microimplants too is imperative to avoid the possibility of root damage and for suitable force vector planning. The complications resulting due to positioning errors of miniscrew implants have been documented. ${ }^{1}$ The problems are:

- Risk of damaging the roots or the periodontium

- Possibility of miniscrew-root contact resulting in early screw failure.

To avoid these risk factors numerous guides and stents with the help of radiographic method have been developed keeping these factors in mind to avoid potential complications. ${ }^{2-5}$ Although the radiographic method is more reliable than clinical observation because it is unaffected by tooth movement, the disadvantage of radiographic measurement is the difficulty of interpreting and applying the results in clinical practice. Thus, accuracy is mandatory for implant placement. A simple, reliable and accurate method for placement of implants in a single step without any complications is described by the authors.

\section{APPLIANCE FABRICATION}

The three-dimensional (3D) acrylic wire grid consists of two parts, a positioning grid (gauge) and a guide base. The positioning grid is fabricated from 24 gauge straight stainless steel wire. The stainless steel wire is cut in to pieces of 1 inch in length and welded to form a column grid, in which each cell measures about $1.5 \mathrm{~mm}^{2}$. The column grid is welded to round ' $U$ ' frame support arm of the positioning grid (Fig. 1A), which is made up of $1 \mathrm{~mm}$ round stainless steel wire. Fabricate a stent base by bending 18 gauge bore round stainless steel needle forming one end to support the grid and securely embedding the other end in cold cure acrylic forming the occlusal surface base (Fig. 1A). The appropriate vertical length of the grid is adjusted according to the desired miniscrew insertion point (generally 5-6 mm apical to the alveolar crest) by moving grid up down in to needle.

Add soften wax on acrylic base (on occlusal side) and press it gently on occlusal surface. After fitting two parts of the 3D acrylic wire grid in mouth (Fig. 1B), take IOPA to show the position of the roots in relation to the stent (Fig. 2). On the radiograph, determine the point between the two adjacent roots, based on the wire grid welded to the horizontal arm of the positioning gauge. Once the appropriate cell of the grid for the exact miniscrew site is selected on the periapical radiograph, the pilot drilling is performed with the grid in place (Fig. 3). After performing pilot drilling then inserts the miniscrew in place (Fig. 4). Use occlusal mirror to visualize the occlusal surface to guide the implant driver (keeping it parallel with directional guide). The grid is then disengaged from the mouth and post placement radiographs can be taken to verify the interradicular placement of miniscrew (Fig. 5). Once the procedure on one side is over, the wax is removed and fresh wax is added on the acrylic base to use it on other side (Figs 6A and B).

\section{Advantages}

1. The guide described is universal.

2. It can be used on any implant insertion site (upper/lower/ ant./post.). 


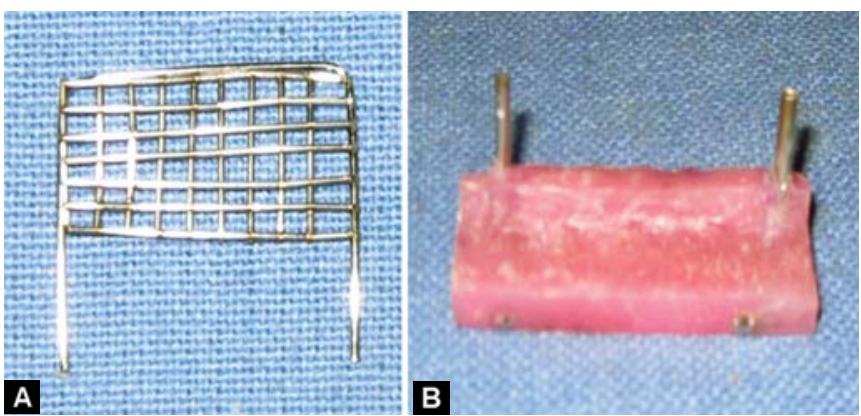

Figs 1A and B: (A) Fabrication of the column grid and welded to round $U$ frame support arm of the positioning grid. Fabricating a stent base by bending 18 gauge bore round stainless steel needle forming one end to support the grid and securely embedding the other end in cold cure acrylic covering the occlusal surface, (B) fit two parts of the $3 \mathrm{D}$ acrylic wire grid in mouth

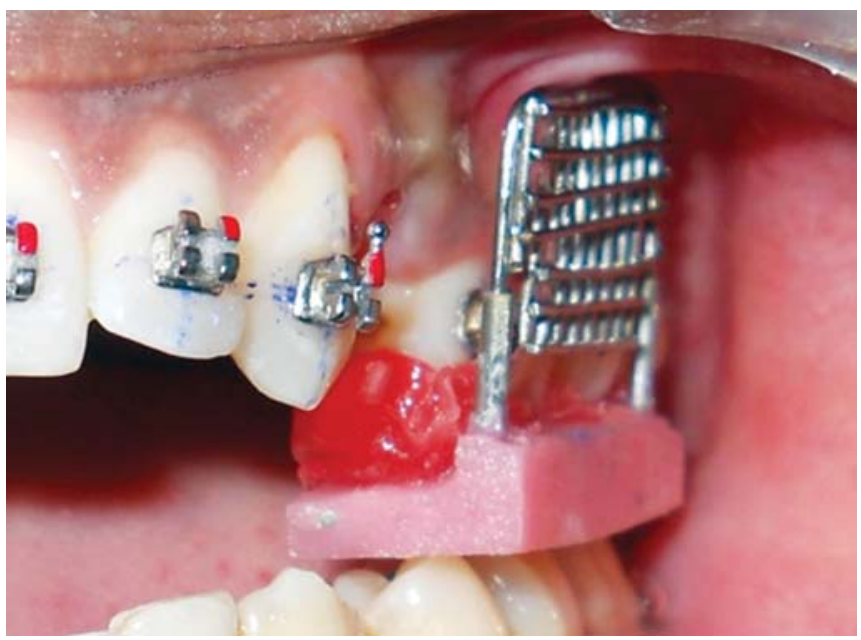

Fig. 2: After fitting into the mouth periapical radiograph showing the position of the roots in relation to the stent

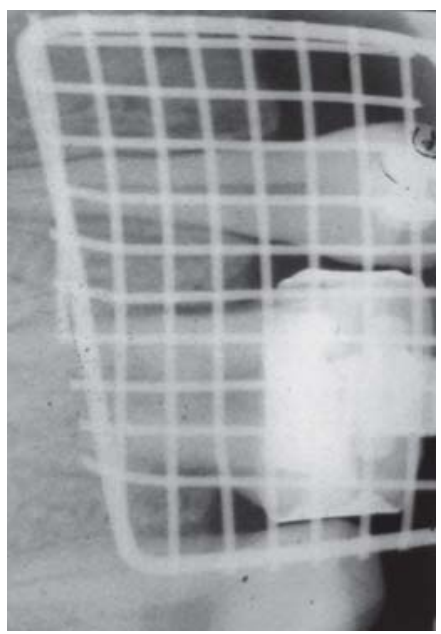

Fig. 3: The pilot drilling is performed with the grid in place

3. After proper sterilization, it can be used in other patients.

4. The procedure described is easy to master.

5. It can be also used for placement of prosthetic implant.

\section{CONCLUSION}

The cortical bone appears to be one of the best sites for orthodontic miniscrews or palatal implants. Cortical

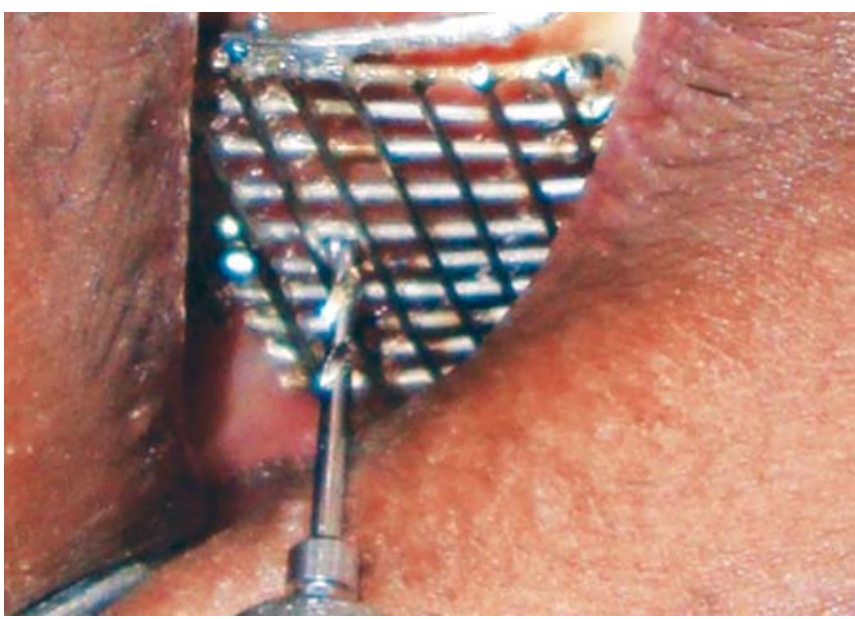

Fig. 4: Insertion of the miniscrew in place along with grid

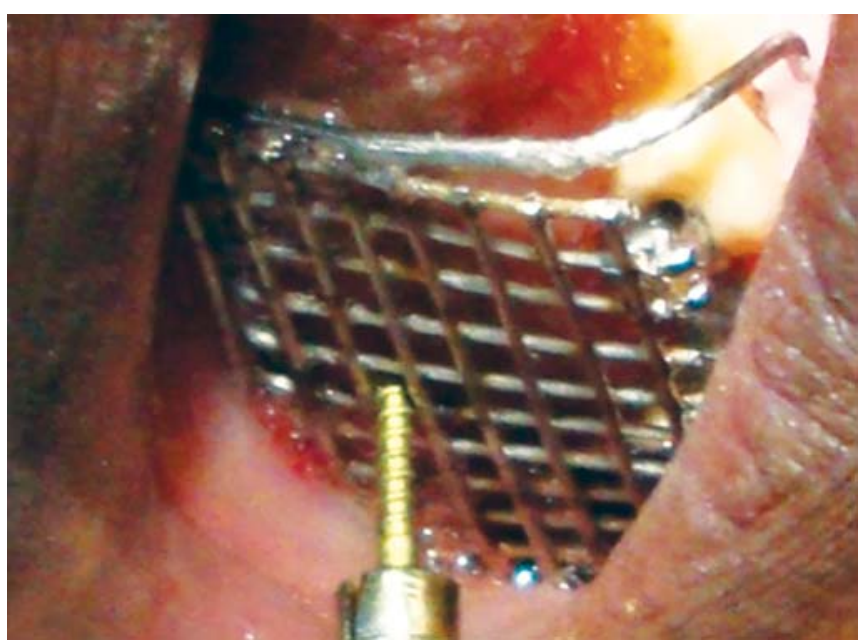

Fig. 5: Post placement radiographs showing the interradicular placement of miniscrew

bone is typically thicker in the palate than at buccal interradicular insertion sites, and favorable attached gingiva is readily available, ensuring high success rates. Miniscrews placed in interradicular area accurately will not contact dental roots or inhibit tooth movement. Skeletal anchorage from miniscrews isoptimal for supporting various treatment mechanics, including distalization, protraction of buccal teeth, rapid maxillary expansion, space closure, and intrusion mechanics. Biomechanics can be designed in nearly any direction and can usually be changed in midtreatment using the same anchorage setup. The alveolus between the roots of the second premolar and first molar may be considered as an ideal miniscrew location, with some limitations.

\section{REFERENCES}

1. Kravitz ND, Kusnoto B. Risks and complications of orthodontic miniscrews. Am J Orthod Dentofacial Orthop 2007;131:43-51.

2. Wu JC, Huang JN, Zhao SF, Xu XJ, Xie ZJ. Radiographic and surgical template for placement of orthodontic microimplants in interradicular areas: A technical note. Int J Oral Maxillofac Implants 2006;21:629-34. 


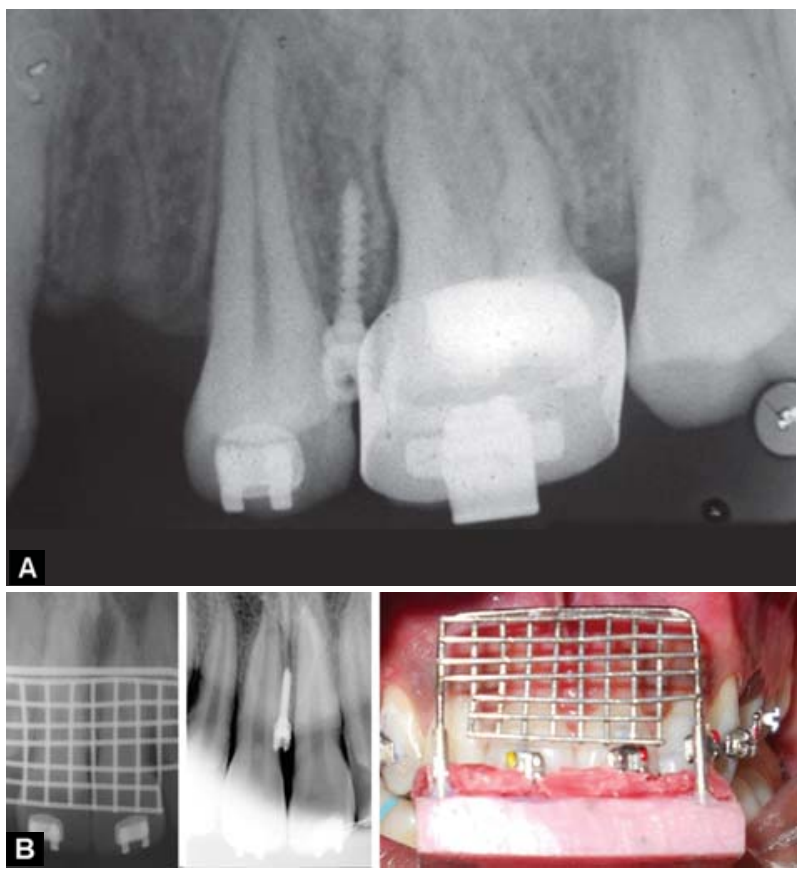

Figs $6 \mathrm{~A}$ and $\mathrm{B}$ : Use on other implant insertion site (upper/lower/ant/post)

3. Suzuki EY, Suzuki B. A simple three-dimensional guide for safe miniscrew placement. J Clin Orthod 2007;41:342-46.

4. Kravitz ND, Kusnoto B, William F, Hohlt WF. A simplified stent for anterior miniscrew insertion. J Clin Orthod 2007;41:224-26.
5. Choi HJ, Kim TW, Kim HW. A precise wire guide for positioning interradicular miniscrews. J Clin Orthod 2007;41: 258-61.

\section{ABOUT THE AUTHORS}

\section{Narendra S Sharma (Corresponding Author)}

Associate Professor, Department of Orthodontics, Sharad Pawar Dental College, Sawangi, Wardha, Maharashtra, India e-mail: sharmanarendra047@gmail.com

\section{Sunita S Shrivastav}

Professor, Department of Orthodontics, Sharad Pawar Dental College Sawangi, Wardha, Maharashtra, India

\section{Pushpa V Hazarey}

Professor and Head, Department of Orthodontics, Sharad Pawar Dental College, Sawangi, Wardha, Maharashtra, India

\section{RH Kamble}

Professor, Department of Orthodontics, Sharad Pawar Dental College Sawangi, Wardha, Maharashtra, India

\section{Preethi N Sharma}

Resident (1st year), Department of Oral Pathology and Microbiology Sharad Pawar Dental College, Sawangi, Wardha, Maharashtra, India 